\title{
LA IMPORTANCIA DE LA INVESTIGACIÓN BÁSICA EN TISP. Una Puerta Entreabierta Para el AVAnCE DEL CONOCIMIENTO
}

\author{
Carmen Valero-Garcés \\ University of Alcalá, \\ Spain \\ carmen.valero@uah.es \\ Bianca Vitalaru \\ University of Alcalá, \\ Spain \\ aula.traduccion@uah.es
}

\begin{abstract}
La investigación es fundamental para el desarrollo de una sociedad. La realidad nos muestra que las sociedades actuales avanzan desde sociedades monoculturales a otras multiculturales, y ello conlleva una serie de cambios y adaptaciones que necesitan ser investigados a fin de encontrar soluciones que faciliten la consolidación de las nuevas estructuras. Cuando están en juego lenguas y culturas diferentes, la presencia de traductores e intérpretes es fundamental. Si además concentramos nuestra atención en las relaciones entre las instituciones u organismos públicos y los inmigrantes, estaremos hablando de traductores e intérpretes en los servicios públicos. El objetivo de este artículo es dar cuenta de las investigaciones empíricas llevadas a cabo por alumnos del Master Universitario en Comunicación Intercultural, Interpretación y Traducción en los Servicios Públicos de la Universidad de Alcalá, cuyos títulos se han incluido en una base de datos ordenada por temas, y proponer acciones de utilidad para el reconocimiento y profesionalización de dicha especialización.
\end{abstract}

\section{Introducción}

Hace apenas un par de décadas que el área que conocemos como "Traducción e Interpretación en los Servicios Públicos" (TISP) comenzó a ganar cierto reconocimiento académico en España, ayudada sin duda por el fenómeno de la inmigración. Estamos todavía muy lejos de llegar al nivel de formación, profesionalización e investigación que han alcanzado otras áreas dentro de los "Estudios de Traducción"; sin embargo, sus avances son claros y se le augura un buen futuro en las sociedades multiculturales hacia las que inevitablemente se camina. En esta ocasión nuestro trabajo tiene un doble objetivo: por un lado, análisis y, y por otro, comentarios y recomendaciones prácticas.

Concretamente, en primer lugar, nos proponemos analizar las dificultades a las que tanto los propios investigadores como los tutores académicos de dichos trabajos se han enfrentado (o se pueden enfrentar) en la elaboración y respectivamente en la dirección de sus investigaciones en el comienzo del siglo XXI cuando se estaba produciendo en España el boom de la inmigración.

En segundo lugar - y contando con la experiencia de la acción formativa en Traducción e Interpretación en los Servicios Públicos iniciada en la Universidad de Alcalá (Madrid, España) en el año 2001- trataremos de extraer las características, tanto formales como de contenido, que presentan las investigaciones llevadas a cabo por estos alumnos procedentes de culturas y lenguas diferentes (más de 15 nacionalidades distintas en cada curso académico). Ello nos permitirá establecer algunas pautas generales como paso previo para la creación de una base de datos que recoja estas investigaciones que modestamente, como formadores, investigadores y practicantes de la TISP, consideramos valiosas. A partir 
de ahí, trataremos de establecer unos criterios generales mínimos y necesarios que pueden significar un paso más en el pleno reconocimiento de la TISP como disciplina académica. Consideramos que también podría constituir un paso más en su profesionalización en esa conjunción de teoría y práctica que subyace como filosofía en todo el proyecto educativo relacionado con lenguas y culturas de la Universidad de Alcalá.

\section{El Máster Universitario en Comunicación Intercultural, Interpretación y Traducción en los Servicios Públicos}

El Máster Universitario en Comunicación Intercultural, Interpretación y Traducción en los Servicios Públicos de la Universidad de Alcalá (UAH), Madrid, se inició como tal en el año 2005, como consolidación de una acción formativa iniciada en el año 2001. El programa desarrollado está en consonancia con el marco del Espacio Europeo de Educación Superior (EEES), dirigido a la búsqueda del equilibrio entre lo académico y lo profesional y la aplicación de un modelo de formación basado en tres pilares fundamentales interconectados: investigación, formación y práctica (figura 1).

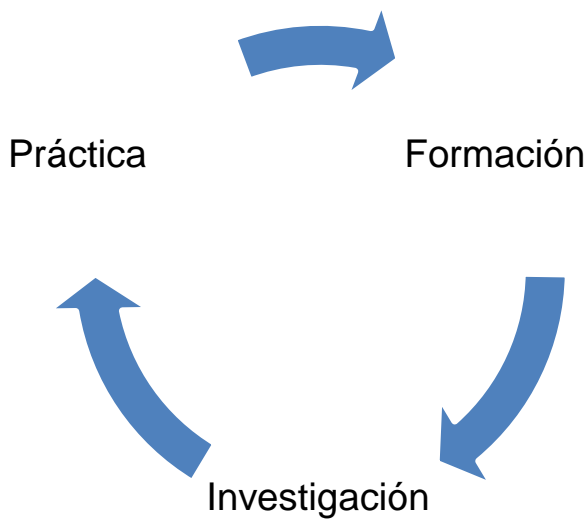

Figura 1. Fundamentos de la formación

Prueba de esta consonancia con los principios del EEES es su integración en 2009 en la Red de Másteres Europeos en Traducción (EU-DGT EMT Network). Ser miembro de la misma le ha permitido unificar objetivos didácticos con los de otras universidades europeas y colaborar en proyectos y acciones como OPTIMALE, (Optimising Professional Translator Training in a Multilingual Europe); AGORA, (Transnational Placement Scheme for Translation Students); QUALETRA (Quality in legal translation) o SIGTIPS (Special Interest Group on Translation and Interpreting in Public Services).

El Máster Universitario en Comunicación Intercultural, Interpretación y Traducción en los Servicios Públicos está organizado en un curso académico. Consta de 60 ECTS (European Credit Transfer and Accumulation System), de los cuales 18 ECTS son comunes a todas las especialidades y 42 ECTS son obligatorios y específicos para cada una de las mismas. Diez son las combinaciones ofrecidas: español y alemán, árabe, búlgaro, chino, francés, inglés, polaco, portugués, rumano y ruso. Dichas combinaciones responden a la 
demanda multilingüe existente en la sociedad española a raíz principalmente de los movimientos migratorios de las últimas décadas.

Los objetivos principales del máster son fundamentalmente dos: por un lado, proporcionar los conocimientos teóricos y las destrezas, habilidades y herramientas necesarias para actuar de enlace lingüístico y cultural entre el personal de las instituciones médicas, jurídicas, administrativas y educativas y los usuarios que no hablan español. Y, por otro lado, dar a conocer y practicar las principales técnicas de este tipo de traducción e interpretación (traducción directa e inversa, interpretación bilateral, consecutiva, traducción a la vista, terminología específica, toma de notas, etc.) en la especialidad lingüística elegida.

El perfil de alumnos al que va dirigido el Master se podría resumir en varias líneas: licenciados o graduados con un conocimiento profundo tanto del español como de al menos otra lengua de las ofrecidas, que han hecho o hacen de enlace con población extranjera para eliminar barreras en situaciones diversas o que cuentan con experiencia como mediadores lingüísticos a nivel oral o escrito (intérpretes y traductores). Como criterios de selección se utilizan, con carácter general y como requisito imprescindible el dominio de las lenguas de trabajo (nivel C1 - C2 según el Marco Europeo de Referencia), seguidos de la formación y la experiencia del estudiante.

La metodología utilizada parte de un enfoque integral y multidisciplinar basado en la aplicación de un modelo multilingüe y multicultural que combina teoría y práctica desde varios ángulos como son:

- Combinación de la enseñanza tradicional en el aula con las nuevas tecnologías y la enseñanza online.

- Participación de destacados profesionales y académicos de diferentes lenguas y culturas así como de representantes de diversas instituciones en debates, ponencias y talleres reducidos según el par de lenguas elegido.

- Participación activa de los alumnos mediante el intercambio de experiencias relacionadas con la traducción e interpretación en distintos ámbitos.

- Práctica en el aula con textos auténticos y en situaciones simuladas basadas en experiencias reales.

- Prácticas en instituciones públicas y privadas que trabajan con población extranjera como introducción al mundo laboral.

- Introducción a la investigación con la realización de un Trabajo fin de máster (TFM) o Memoria de máster sobre un tema relacionado con la temática de la acción formativa que capacita al alumno para la búsqueda de documentación, preparación de proyectos y colaboración con colectivos de diversas instituciones.

A lo largo de todo el curso, los alumnos reciben asesoramiento y ayuda a través de diferentes tipos de tutorías estructuradas y diferenciadas, como son las tutorías académicas con los profesores del máster y con los coordinadores de la especialidad, las tutorías sobre el Trabajo fin de máster y las Tutorías de Prácticas. En éstas últimas cada alumno tiene un tutor de prácticas en la Universidad y un tutor en el centro externo en el que realiza sus prácticas externas.

El plan de estudios está dividido en cinco módulos, cada uno de ellos con una serie de asignaturas, tal y como se muestra a continuación:

1. Módulo I: Comunicación intercultural e interlingüística (módulo online común), que se compone de tres asignaturas:

Comunicación interlingüística (5 ECTS)

Comunicación institucional con población extranjera (7 ECTS)

Técnicas y recursos para la TISP (online y presencial) (6 ECTS) 
2. Módulo II: Traducción e interpretación sanitaria (clases presenciales, según la especialidad elegida), que se compone de dos asignaturas:

Interpretación en el ámbito sanitario (5 ECTS)

Traducción especializada: ámbito sanitario (5 ECTS)

3. Módulo III: Traducción e interpretación jurídico-administrativa (clases presenciales, según la especialidad elegida), que se compone de tres asignaturas:

Interpretación en el ámbito jurídico - administrativo (8 ECTS)

Traducción especializada: ámbito jurídico (5 ECTS)

Traducción especializada: ámbito administrativo (5 ECTS)

4. Módulo IV: Prácticas en empresas e instituciones (5 ECTS)

5. Módulo V: Trabajo fin de máster/ Memoria de máster (9 ECTS).

En este artículo nos centraremos en este último módulo - el Módulo V- cuyo objetivo es la realización de un trabajo de investigación. Con dicho trabajo se pretende que el alumno se inicie en la investigación y sepa exponer sus resultados ante un tribunal. De acuerdo con las normas que rigen los TFM, éstos deben tener un carácter fundamentalmente práctico basado en investigación empírica y con una extensión entre 40.00050 .000 palabras. El tema del trabajo debe estar relacionado con la TISP, bien elegido entre los propuestos por la dirección del master, bien propuesto por el alumno. En ambos casos, y al tratarse de un tema individual, deberá ser aprobado por la dirección del master, que le asignará un tutor en función del tema y de las lenguas de trabajo.

Completado el TFM, la valoración del trabajo escrito se lleva a cabo por dos profesores. Uno es el tutor que le ha estado dirigiendo y el otro es elegido entre los formadores del curso, atendiendo a criterios de afinidad de tema, lengua, perfil del alumno o tipo de investigación (empírica, teórica, de aplicación práctica, etc.). Si la parte escrita es aprobada, pasa a la defensa oral ante un tribunal compuesto por tres miembros. Superada esta fase, se da el TFM por aprobado.

\subsection{Puntos negros en la investigación en TISP}

En el contexto del programa formativo cabe destacar la utilidad del tipo de investigación propuesto en este artículo, ya que, como indica Cohen et al. (2000: 226), parafraseando a Lewin, autor del término "investigación-acción"), "Research which produces nothing but books is inadequate", es decir la investigación que no produce nada más que libros es insuficiente. Consideramos pues, que, si la investigación ha de tener alguna utilidad en el campo de la traducción e interpretación en los servicios públicos, es necesario que ésta sea práctica y accesible. De ahí que uno de los objetivos propuestos sea dar a conocer la investigación realizada por nuestros alumnos a través de los TFM.

Todo ello implica, pues, un nuevo reto. Tal y como indica Hale (2010: 204), aún siendo la investigación práctica y relevante, su accesibilidad es todavía un reto. Hale comenta que gran parte de la investigación dentro de la interpretación en los servicios públicos (o comunitaria) es práctica ya que la han dirigido investigadores que todavía trabajan como intérpretes o que tenían una amplia experiencia como intérpretes antes de convertirse en investigadores académicos. En el caso de la traducción la situación sería similar si bien la cantidad de publicaciones es menor y quizás haya sido producida más por académicos, punto que nos queda por investigar en el futuro. 
No obstante, muchos de los practicantes en activo así como de los proveedores de estos servicios (sean las propias instituciones $\mathrm{u}$ organizaciones o los individuos) con frecuencia piensan que la investigación no tiene ninguna importancia para ellos, lo que quizás se deba a que no leen material de investigación o no ven un aplicación directa de las conclusiones en su día a día. Además, muy pocos profesionales de la TISP, y menos si no lo son de este ámbito, tienen el tiempo, el saber hacer o la disposición para buscar y leer bibliografía de investigación. Se plantea así un reto a los investigadores: cambiar esta actitud negativa haciendo que los resultados de sus investigaciones sean más accesibles.

Centrándonos específicamente en el tema que nos ocupa, los 438 Trabajos de fin de máster (TFM) entregados hasta marzo de 2014 e incluidos en nuestra base de datos constituyen, sin duda, una buena fuente de conocimiento y contribución al desarrollo y profesionalización de la TISP. En ellos se puede observar variedad de métodos y, corroborando las palabras de Hale, grosso modo, podemos decir que hay más investigación aplicada que teórica, con trabajos que cabría clasificar como exploratorios, descriptivos, e incluso como explicativos, sobre todo en las últimas ediciones. A través de ellos observamos la evolución de la sociedad, española cuando menos y, nos atreveríamos a decir, internacional, por las características del alumnado- investigador. Los TFM nos permiten comprobar lo que otras fuentes nos muestran: la formación de una sociedad multicultural y multilingüe, la llegada masiva de lenguas y culturas en décadas pasadas, las nuevas necesidades surgidas en la comunicación, las soluciones que se van dando y la etapa de consolidación que parece acercarse. Dicha consolidación pasa por el conocimiento de los unos y los otros, y la investigación es parte fundamental de ese proceso. Tarea no fácil ante las barreras que la dificultan en España y que resumimos a continuación (Valero Garcés 2013a: 80-81, b: 217-18).

1. Falta de concienciación o interés por la comunicación de calidad en entornos institucionales $\mathrm{u}$ organismos acostumbrados a un modelo fundamentalmente monolingüe.

2. La barrera lingüística como cortapisa a la investigación, al no disponer los investigadores de materiales o no tener acceso a investigaciones que están principalmente en inglés. Ello supone un obstáculo para muchos de estos estudiosos de lenguas como el árabe, búlgaro, rumano, polaco, ruso o chino que han aprendido la lengua del país de acogida (español en este caso), pero desconocen el inglés.

3. Las diferencias en el acceso y uso de las nuevas tecnologías aplicadas a la investigación y la comunicación (TIC). En el caso de las lenguas llamadas "minoritarias" aún falta mucho por hacer: desarrollo y adaptación de programas de traducción automática, creación de bases de datos, redes internacionales, bancos multilingües en las lenguas minoritarias o combinaciones entre lenguas que no eran frecuentes hasta este siglo, por citar algunos ejemplos.

4. La falta de reconocimiento (o desconocimiento) de la actividad traductora y de interpretación profesional como elementos clave para la comunicación rápida y efectiva. Ello a pesar de los resultados de estudios llevados a cabo en países con una mayor tradición en la recepción de población inmigrante en cuanto a la eliminación de costes y ahorro de tiempo que un servicio de traducción e interpretación adecuado conlleva (Grubbs et al 2006, Jacobs et al 2004, Morales et al 2006, Sturby 2007).

5. La falta de organización y control de calidad en la explotación de recursos disponibles. Ello contribuye aún más a dificultar la comunicación. Así, por ejemplo, la incorporación de materiales multilingües exige en muchos casos una labor de revisión de su calidad, tanto de los materiales traducidos y distribuidos en papel como de los disponibles en la red, y no sólo a lenguas minoritarias con respecto a cada país 
sino también a lenguas más conocidas. Se necesita también una labor de localización para adaptar dichos materiales a la situación concreta en la que se van a utilizar o bien para crear nuevos materiales en la lengua meta (LM), dirigidos a un público específico. Sin embargo, esa labor, según nuestros datos, no se está llevando a cabo hoy por hoy.

6. La falta de estudios interdisciplinarios y de la unión de esfuerzos en el avance hacia una investigación de calidad. Hay que eliminar reticencias y confiar incluso- con la precaución que ello requiere- en los trabajos de investigación llevados a cabo por investigadores menos experimentados como pueden ser los autores de estos TFM pero que conocen bien, o forman parte de esas comunidades de inmigrantes. Sus investigaciones son herramientas valiosas, con todas sus deficiencias, para iniciar la construcción de un edificio sólido y una sociedad compacta multicultural.

7. La falta de vías de acercamiento y modelos de integración entre lenguas y culturas que han entrado en contacto recientemente como consecuencia de los movimientos migratorios. De ahí deriva otra razón de peso para apoyar la investigación llevada a cabo por estos alumnos de postgrado: el hecho de que, a veces, este es el único medio efectivo y directo del que se dispone para conseguir adentrarse en el conocimiento de las minorías étnicas y de su cultura, así como de conocer de primera mano problemas concretos (sean comunicativos, educativos, laborales, sanitarios...) o grado de adaptación. Este tipo de conocimiento es importante para conseguir una sociedad multicultural considerando también la circunstancia favorable de que, a veces, los alumnos pertenecen a dichas comunidades y les resulta, por tanto, más fácil penetrar en ellas.

8. Dificultad de acceso a la investigación empírica y la obtención de datos por las barreras que con frecuencia ponen las instituciones o la administración alegando cuestiones- en muchos casos injustificadas- de privacidad u otros motivos que impiden el acceso a dichos datos, lo cual condiciona el número relativamente bajo de datos que encontramos en los trabajos. Esto evidencia, una vez más, el escaso apoyo a la investigación.

9. La falta de rigor en el procesamiento de los datos es también un factor a tener en cuenta al carecer, con frecuencia, de formación en el uso de herramientas de investigación tanto cualitativa como cuantitativa.

10. El perfil de los investigadores y tutores. Ya hemos mencionado su diversidad cultural y lingüística. A ello hay que añadir, tal vez como una consecuencia más, cierta falta de madurez investigadora en algunos alumnos relacionada, entre otras causas, con sus países de procedencia al tener sistemas educativos diferentes y además enfrentarse a una cultura y lengua alejada de su lengua materna. Ello exige también una mayor dedicación por parte de los tutores, los cuales con frecuencia provienen también del entorno profesional más que académico. Dicha carencia se ve, no obstante, compensada en ambos casos por la información que contienen sus trabajos como elementos clave para progresar en ese conocimiento mutuo unido a las orientaciones prácticas que añaden los tutores desde su propia experiencia.

\subsection{Puntos blancos en la investigación en TISP}

En cuanto al valor de esta investigación cabría mencionar algunos puntos clave:

1. La importancia de la investigación de los TFM para el conocimiento de las nuevas comunidades y la formación de una sociedad plural.

2. La variedad de títulos e iniciativas que contienen referidas a áreas diversas (jurídica, 
médica, educativa, administrativa).

3. La validez de algunos trabajos como primeros pasos para avanzar en el conocimiento.

4. La incorporación de las nuevas tecnologías en combinaciones lingüísticas en las que no hay aún materiales de ese tipo, lo cual indica añadir un grado de actualidad.

5. La aportación de soluciones prácticas y realistas a cuestiones concretas en una cultura y lengua específicas en relación con la cultura mayoritaria.

6. La búsqueda de bibliografía o recursos concretos en otras lenguas que son traidos al español o a la inversa, la puesta en la otra lengua de materiales en español o en otras lenguas.

7. La aportación de materiales útiles y auténticos para la formación derivados de los datos obtenidos o corpus usados en su investigación.

8. La contribución a la mejora del programa de formación al mostrar la realidad exterior y la preparación que la misma o los proveedores exigen.

9. El acercamiento a los profesionales de materiales y bibliografía o recursos como pequeñas píldoras hechas accesibles en los seminarios de formación o jornadas que el grupo FITISPos periódicamente organiza (ver http://www2.uah.es/traduccion ).

10. La labor de difusión y acceso al público de investigaciones específicas muy poco conocidas o prácticamente desconocidas al no estar publicadas.

\section{Acciones de utilidad para el reconocimiento y profesionalización de dicha especialización}

Llegamos así al último punto de nuestro artículo: proponer acciones de utilidad derivadas de la investigación contenida en los TFM para que los esfuerzos puestos en ellos no queden en un cajón cerrado y poder reclamar así su valor.

En este sentido, hay ya en marcha, o a punto de iniciarse, una serie de acciones derivadas de estas investigaciones con el objetivo de divulgar estos trabajos y promover el interés por la comunidad científica y por los futuros traductores e intérpretes a los que animamos a materializar su esfuerzo en una tesis doctoral o en estudios más profundos sobre temas aún por explorar.

Tres de estas acciones son precisamente la razón de este artículo que esperamos sirva como presentación de las mismas:

1. Creación de un catálogo-repositorio de títulos de Trabajos de fin de máster

2. Análisis del contenido de la base de datos de TFM y recopilación de datos específicos por temática y especialidad lingüística.

3. Publicación periódica de trabajos derivados de los TFM en la revista científica FITISPos International Journal.

Y a ello, cabe añadir un punto más, 4, con otras medidas de promoción del trabajo en TISP.

\subsection{Creación de un catálogo-repositorio de títulos de Trabajos fin de máster}

Ya creado desde hace más de 3 años y actualizado en cada curso académico, el equipo investigador del máster reseñado cuenta con un catálogo-repositorio de títulos de Trabajos de fin de máster compuesto de una serie de archivos o compartimentos que incluyen conjuntos de fichas ordenadas según su temática e identificadas por el título, nombre del autor, año de defensa del TFM, una serie de palabras clave y un resumen en inglés y en español. En este punto cabe destacar el número total de trabajos realizados hasta ahora (438) así como el 
número por lenguas durante cada curso académico para poder hacernos una imagen global de la cantidad de información que se ha incluido en el catálogo. De un total de 438 trabajos, 162 se han realizado en la especialidad inglés-español (37\%), 81 en chino-español (18\%), 69 en francés-español (16 \%), 39 de árabe-español (9 \%), 34 en rumano-español (8\%), 32 en ruso (7\%) y 21 en polaco-español (5\%) (ver figura 2$)$.

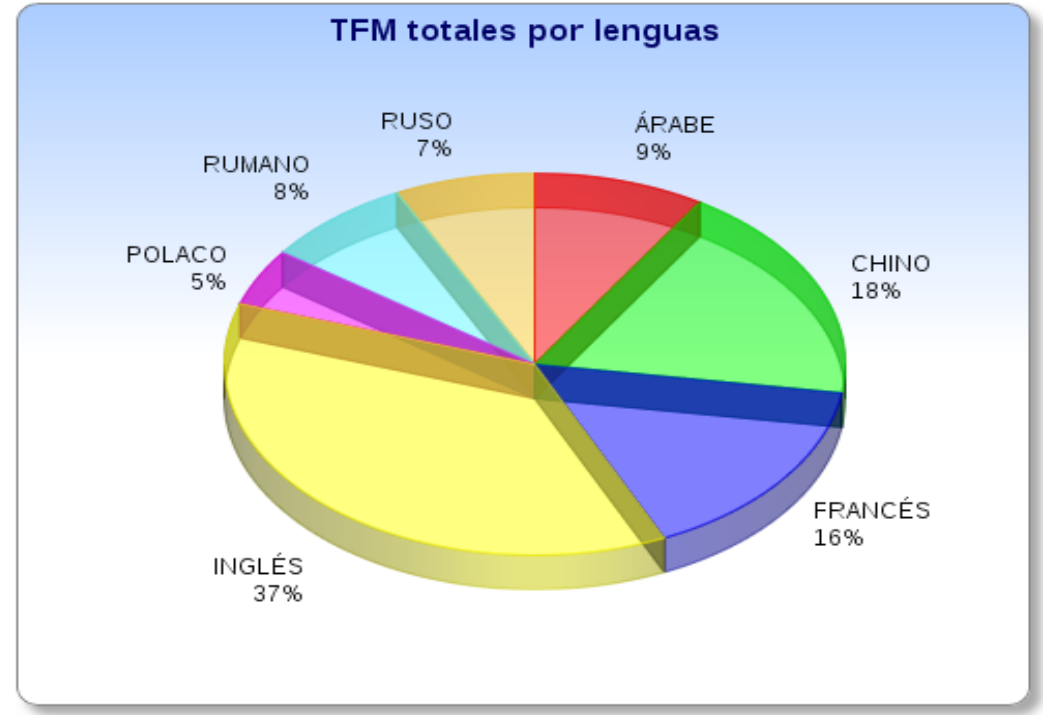

Figura 2. TFM totales por lenguas cursos académicos 2006-2013

Este catálogo-repositorio está actualmente disponible para los alumnos del Máster Universitario en Comunicación Intercultural, Traducción e Interpretación en los Servicios Públicos a través de la plataforma virtual (campus online) de la Universidad de Alcalá. Para un acceso más fácil y rápido, las fichas bilingües completas de cada TFM, que incluyen una serie de datos importantes como autor, tutor, palabras clave y resumen, están organizadas por lenguas y cursos académicos.

3.2. Análisis del contenido de la base de datos de TFM y recopilación de datos específicos por temática y especialidad lingüística.

En el punto 3.1 ya mencionábamos el número de trabajos realizados por especialidades linguísticas, datos que nos ayudan en la clasificación como punto de partida en el momento de llevar a cabo esta investigación. En este punto (3.2) nos centraremos en un análisis más específico de los datos considerando tres factores: temática, especialidad linguiística y curso académico.

En cuanto a la temática de los mismos, tras un estudio de los TFM evaluados (un total de 438), en un intento inicial de clasificación, se agrupan en seis bloques generales (Valero Garcés 2008, 2012), si bien hay que admitir constantes solapamientos y dificultades para establecer líneas divisorias claras entre uno y otro bloque:

1. Estudios sobre el estado de la cuestión

2. Estudios sobre modos de comunicación intercultural

3. Estudios sobre formación en TISP

4. Traducción, adaptación y/o producción de textos en otras lenguas

5. Creación de recursos y terminología

6. Estudios sobre aspectos teóricos. 
Un análisis más detallado nos lleva a establecer hasta 19 categorías según temática, considerando su utilidad desde el punto de vista formativo e investigador. Éstas son:

- Análisis de materiales traducidos

- Aspectos culturales y mediación. Comunicación intercultural

- Diccionarios y guías

- Elaboración de materiales

- Estudios comparativos

- Estudios descriptivos

- Género

- Impacto psicológico

- Interpretación de signos

- Interpretación telefónica/remota

- Papel del traductor e interprete

- Profesionalización de la traducción e interpretación

- Programas de formación en TISP

- TISP y medios de comunicación

- Toma de notas y memoria

- Traducción e interpretación en ámbito educativo

- Traducción e Interpretación en ámbito jurídico

- Traducción e Interpretación en ámbito sanitario

- Traducciones comentadas de textos.

En en siguiente gráfico se presentan las categorías mencionadas con su número correspondiente de trabajos realizados en cada tema (ver figura 3):

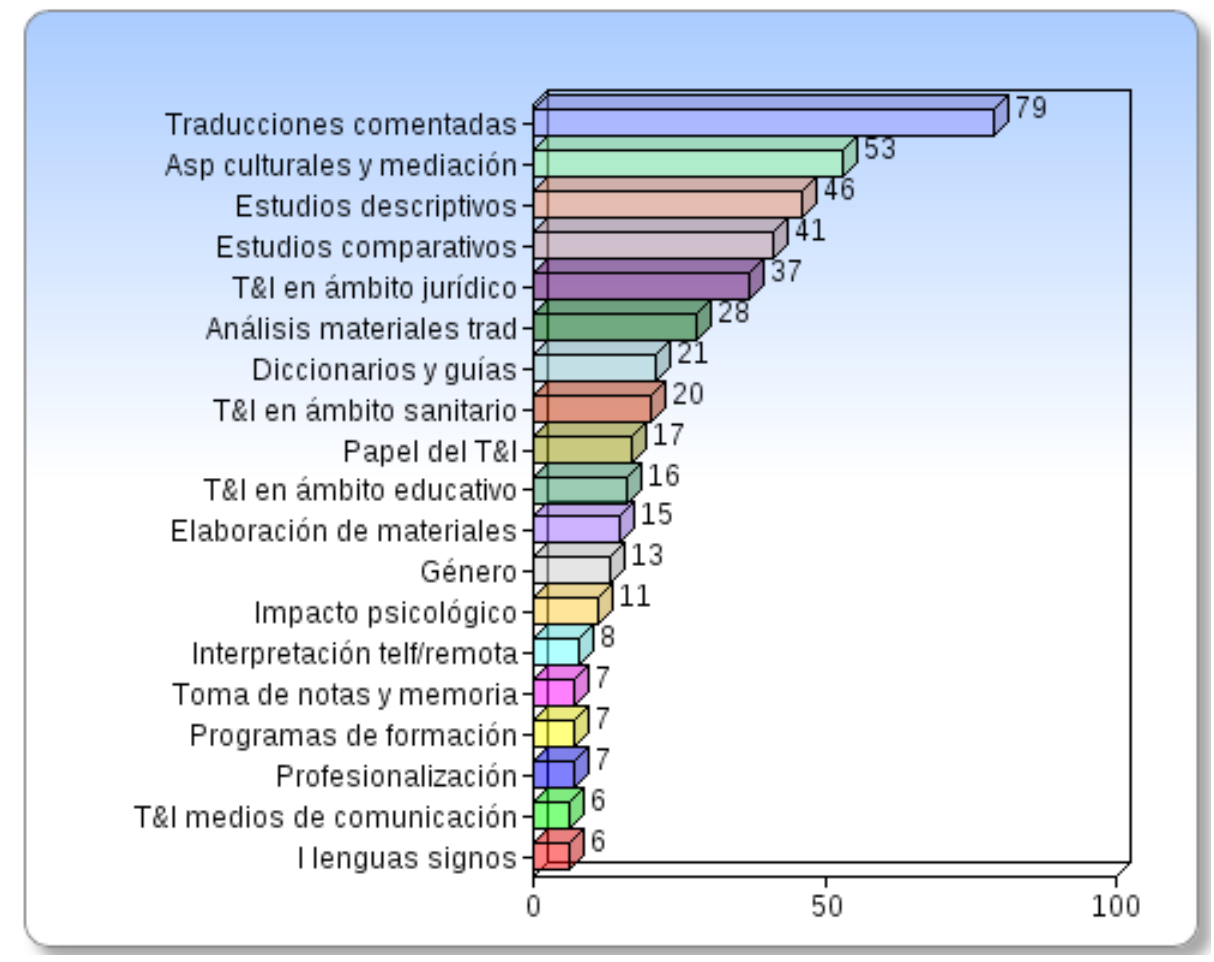

Figura 3. Categorías temáticas TFM catálogo-repositorio

Un análisis específico muestra el alto porcentaje de TFM de traducción comentada $(18,03 \%)$ del número total de TFM considerando todas las especialidades lingüísticas. De ellas, el 31,64 \% se han realizado en la combinación inglés-español y 20,25\% en la 
combinación chino-español. Esto se debe principalmente al gran número de matriculados en estas dos especialidades (aproximadamente 35 alumnos en los dos últimos cursos académicos), así como al interés por la traducción a pesar de tratarse de un análisis de los procesos de investigación, documentación y traducción y no de una mera traducción en sí. En cuanto al tipo de textos traducidos son principalmente artículos científicos y actas de congresos sobre temas relacionadas con la TISP, guías y libros sobre mediación, comunicación intercultural y aspectos sanitarios o incluso legislación específica del ámbito jurídico.

En segundo lugar, remarcamos el gran interés por la comunicación intercultural, los aspectos culturales y la mediación, que ocupa un 12,10 \% del total de los TFM. Este tema se ha tratado especialmente en los cursos 2009-10, 2011-12 y 2012-13, en las especialidades chino-español, francés-español e inglés-español (considerando las variedades de otros países en las que se hablan estos idiomas). En la especialidad árabe-español, donde se trabaja con culturas muy distintas, el número de trabajos ha sido bastante bajo $(9,43 \%$ de un total de 53 trabajos sobre este tema) mientras que en las especialidades rumano-español y ruso-español el número ha sido similar $(9,43 \%$ y $7,54 \%)$ aunque en estos últimos dos casos se debe a la cercanía cultural entre estos países y al número más reducido de diferencias culturales.

En tercer lugar, observamos un gran interés por los estudios descriptivos sobre la situación de la TISP $(10,50 \%)$ o comparaciones de varios países $(9,36 \%)$, sobre todo en las primeras ediciones. Tal interés parece lógico si consideramos que es el único master de estas características ofrecido en España en las especialidades o combinaciones lingüísticas ya mencionadas, pero que cuenta con alumnos de más de 15 nacionalidades cada año. Además, un factor importante es la escasez o inexistencia de trabajos sobre TISP relacionados con España y con el resto de los países.

Existe igualmente un alto interés $(6,39 \%)$ por conocer la existencia de materiales multilingües o traducidos a las respectivas lenguas, así como una evidente preocupación por la calidad de los mismos, aspecto igualmente comprensible teniendo en cuenta que son traductores e intérpretes en formación y uno de los principios de la enseñanza que reciben se basa en la calidad.

En los últimos cursos académicos los alumnos se han orientado hacia la elaboración de materiales y guías $(3,42 \%)$ así como de glosarios, diccionarios y herramientas de gestión terminológica $(4,79 \%)$, especialmente en especialidades linguiísticas que carecen de 0 cuentan con muy pocos recursos especializados bilingües como el polaco, el chino y el rumano-español (cada uno representando un 19,44 \% de un total de 36 trabajos) y el rusoespañol $(11,11 \%)$. En árabe sólo se centraron el 5,55\% mientras que en inglés-español el porcentaje es muy alto $(16,66 \%)$, si consideramos el número total de trabajos de este tipo. A su vez, este número no es muy alto en la especialidad inglés-español $(3,70 \%)$ si consideramos el número total de alumnos de esta especialidad en sí, es decir 6 alumnos de un total de 162.

En cuanto a ámbitos específicos, hemos incluido en categorías separadas los TFM dedicados a los ámbitos sanitario, educativo y jurídico ya que representan, junto con los "Aspectos relacionados con cultura y mediación" y "Terminología y recursos", los ámbitos principales de estudio de nuestro programa de formación. Cada uno tiene un porcentaje bastante alto teniendo en cuenta dos elementos: el número total de trabajos de todos los cursos (figura 4) y el número de trabajos dentro de cada especialidad lingüística (figura 5): 


\section{Porcentaje TFM por ámbitos}

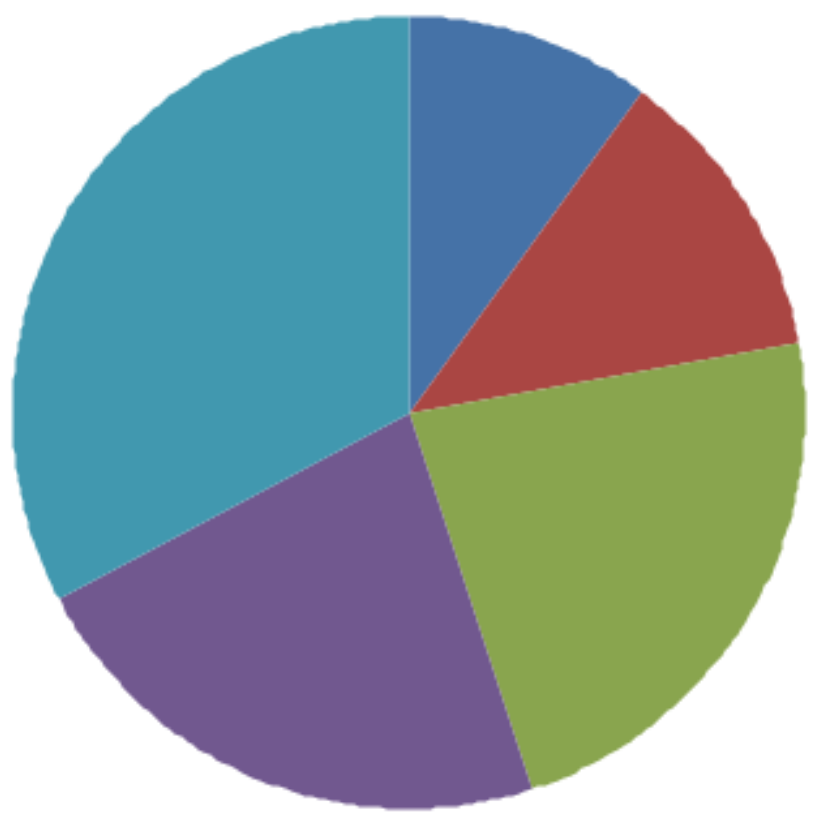

- TFM ámbito educativo

- TFM ámbito sanitario

- TFM ámbito jurídico

- TFM Terminología y

recursos

- TFM Cultura y mediación

Figura 4. Porcentaje TFM ámbitos educativo, sanitario y jurídico

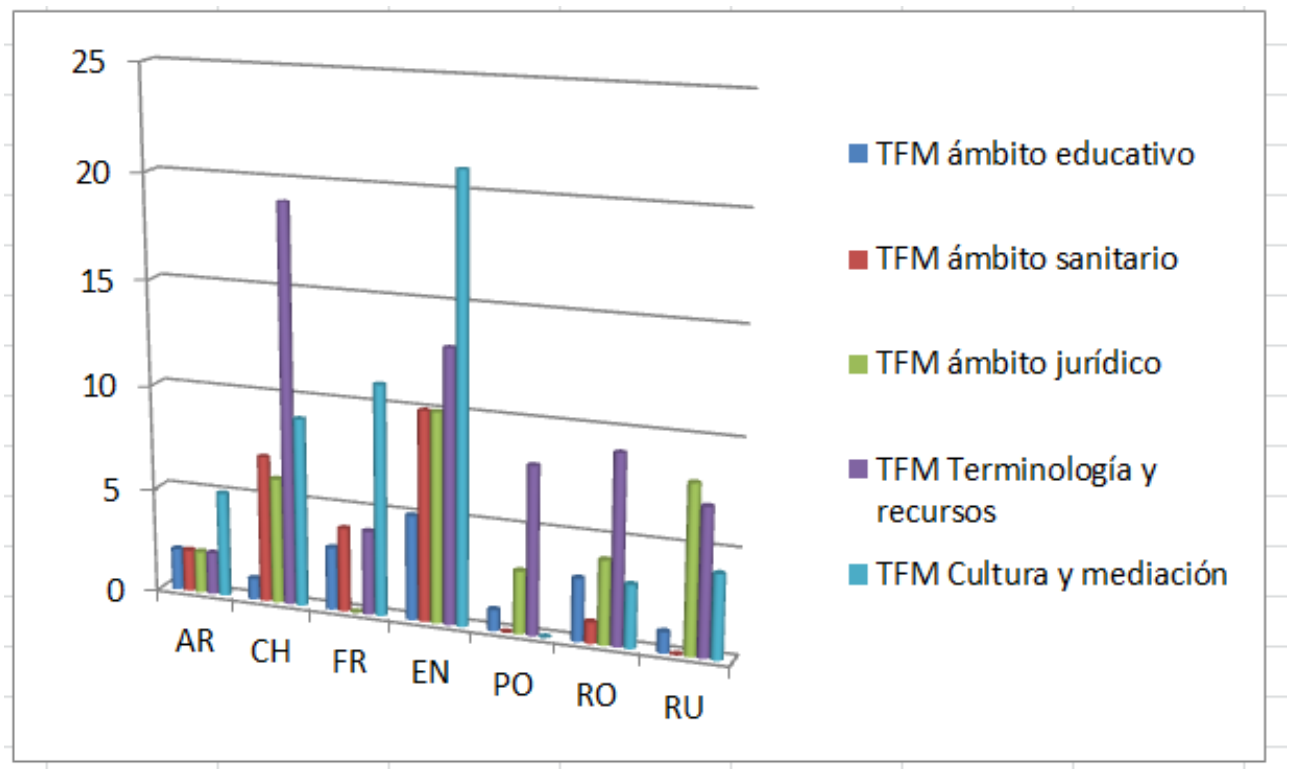

Figura 5. TFM ámbitos educativo, sanitario y jurídico por especialidades

En este sentido, y viendo la figura 4, destacamos que si se considera la totalidad de los TFM, el porcentaje más alto de los tres ámbitos que nos interesa (sanitario, jurídico y educativo) lo tiene el ámbito jurídico $(8,44 \%)$ seguido del ámbito sanitario $(4,56 \%)$ y en tercer lugar el ámbito educativo $(3,65 \%)$.

Sin embargo, si tenemos en cuenta la distribución por especialidades lingüísticas especificadas en la figura 5 observamos variaciones. Por ejemplo, mientras que en las especialidades chino, polaco, rumano y ruso-español es más alto el porcentaje de temas de ámbito jurídico, en inglés-español tenemos el mismo porcentaje para los ámbitos jurídico y sanitario, por encima del ámbito educativo. En la especialidad árabe-español los tres ámbitos tienen el mismo porcentaje. Por otro lado, en la especialidad chino-español el porcentaje de 
TFM de ámbito sanitario es más alto que los demás $(8,64 \%$ del total de TFM de chino frente a 7,40 \% del ámbito jurídico y 1,23\% del ámbito educativo). Lo mismo ocurre en francés con un porcentaje de 5,79\% del total de TFM de esta especialidad para el ámbito sanitario frente al 4,34 \% del ámbito jurídico y $1 \%$ del ámbito educativo. Observamos también el porcentaje muy bajo de TFM de tema sanitario en las especialidades polaco y ruso-español así como del tema educativo para rumano-español.

Por otro lado, debemos tener en cuenta que de estas categorías formarían parte la mayoría de los TFM agrupados en las demás categorías (Interpretación, Papel del Traductor/intérprete, Análisis de materiales, Estudios descriptivos y comparativos, Impacto psicológico, Género, Profesionalización, Programas de formación, Traducción comentada) ya que en cada una se analizan una serie de aspectos dentro de uno o varios de estos ámbitos concretos.

Otra observación interesante que nos gustaría hacer es la existencia de otra categoría significativa que se puede ver en la clasificación: la categoría de TFM relacionados con la interpretación que podría ser subclasificada en otras cuatro subcategorías importantes desde el punto de vista formativo e investigador: Toma de notas; Memoria y procesos cognitivos; Papel del traductor/intérprete, Impacto emocional; Interpretación telefónica y remota. De éstas destaca el porcentaje alto de los TFM sobre Impacto emocional $37 \%$ de un total de 32 de trabajos sobre interpretación) y sobre interpretación telefónica o remota ( $25 \%$ del total de trabajos sobre interpretación) (ver figura 6).

\section{Subcategorías Interpretación TFM}

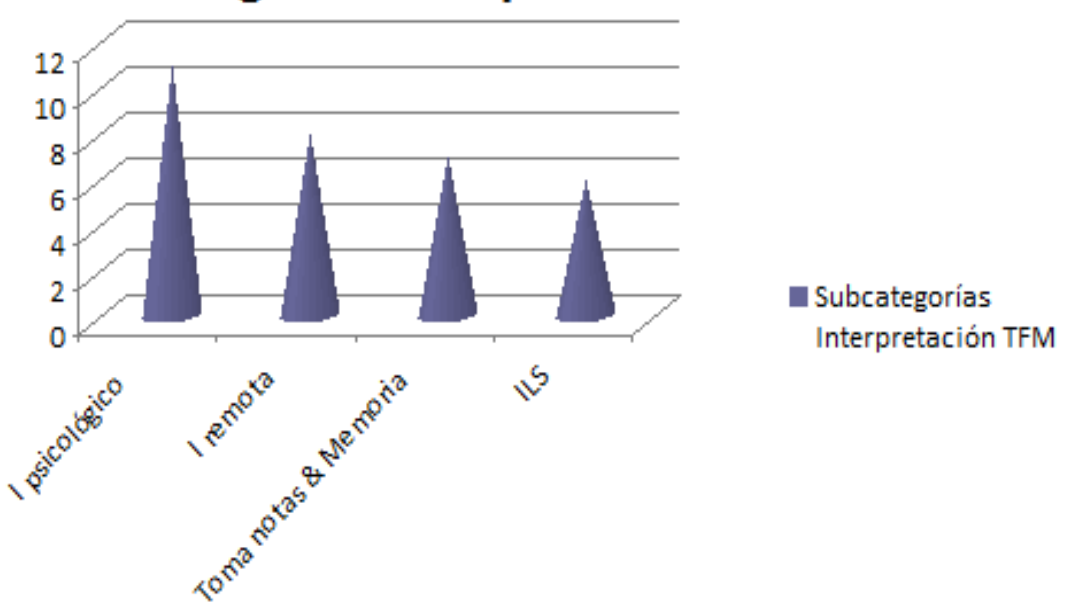

Figura 6. TFM categoría Interpretación

Finalmente, mencionamos otras categorías más específicas incluidas en el catálogo, con un porcentaje más bajo como: Estudios y análisis sobre género, TISP y medios de comunicación, Interpretación de lenguas de signos, Profesionalización de la TISP, Programas de formación en TISP. De éstas la categoría con el porcentaje más alto es la primera con un porcentaje de $3 \%$ del número total de TFM, seguido por la Profesionalización y los Programas de formación en TISP con $1,60 \%$ y por la TISP y medios de comunicación, Interpretación de lenguas de signos con 1,40 \% (figura 7). 


\section{Otras categorías TFM}

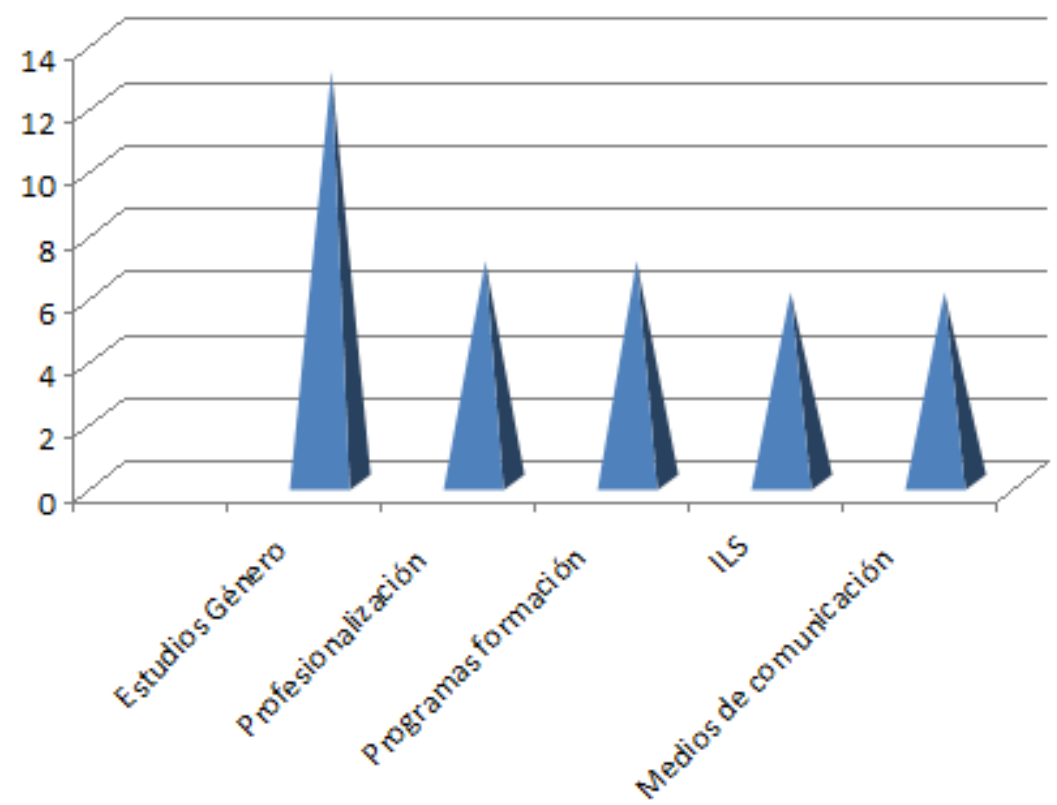

Figura 7. Otras categorías TFM

3.3. Publicación periódica de trabajos derivados de los TFM en la revista científica FITISPos International Journal

El primer número de la revista científica FITISPos International Journal (http://www3.uah.es/fitispos_ij/) ha sido lanzado recientemente (abril 2014) por el grupo de investigación FITISPos). Además de servir de medio para la publicación y difusión internacional de artículos científicos y trabajos de investigación en curso originales relacionados especialmente con la TISP y la Comunicación Intercultural, uno de sus objetivos es precisamente el de dar una oportunidad a futuros traductores e intérpretes para promocionar algunas de las investigaciones más inéditas y de utilidad práctica en las lenguas minoritarias del máster (árabe, chino, francés, inglés, polaco, rumano y ruso). De otro modo, probablemente estas investigaciones se quedarían mucho más tiempo sin darse a conocer.

\subsection{Otras medidas de promoción del trabajo en TISP}

Además de estos tres tipos de acciones ya en marcha existen otra serie de medidas que nuestros propios exalumnos o que desde el grupo FITISPos-UAH se están llevando a cabo con ayuda de colaboradores para contribuir al fomento de la investigación y promoción de nuestro campo de trabajo. Nos centraremos, aunque no detalladamente, principalmente en tres de ellas:

- Profundización en algunos temas a través de la elaboración de tesis

- doctorales.

- Colaboración en la creación de una colección de materiales multilingües.

- Difusión en congresos y revistas.

En primer lugar, cabe mencionar la profundización en algunos temas a través de la elaboración de tesis doctorales. Todas ellas han sido o están siendo elaboradas por alumnos del máster, algunos de ellos colaboradores en la docencia del mismo. Algunas ya están finalizadas y las mencionamos a continuación: Terminología penal y procesal penal: diseño y 
elaboración de un glosario ontológico especializado (español-rumano-inglés) por Bianca Vitalaru o Lingüística cognitiva y traducción de metáforas religiosas (rumano, español) por Aurora Comsa defendidas en el año 2012 y otras tesis en marcha como, por ejemplo, Diseño de un modelo de aplicación para la enseñanza de interpretación de enlace en el ámbito sanitario, por Denis Socarrás; Interpretación en los servicios públicos desde la perspectiva de género por Anca Bodzer; Diseño y compilación de un corpus ad-hoc para la traducción jurídica rumano español por Camelia Hadasa; Investigación sobre el impacto psicológico y emocional en los intérpretes y traductores, por Zuzana Svakova; o Intérpretes y guíasintérpretes de lengua de signos española en servicios públicos de la Comunidad de Madrid: mediadores lingüísticos y culturales, por Úrsula Valenciano; Análisis contrastivo de refranes en chino, español e inglés y su aplicación en la traducción por Hongying Zhang; o Estudio comparativo de la traducción e interpretación judicial en España y Marruecos. Propuesta de elaboración de un diccionario jurídico en materia penal y procesal penal por Sarah Khay.

En segundo lugar, subrayamos la colaboración de una serie de personas en la creación de una colección de materiales multilingües sobre temas específicos relacionados con la TISP: Guías multilingües sobre salud, servicios sociales, o temas educativos en español, árabe, búlgaro, chino, francés, inglés, polaco, rumano y ruso). Estas güías y publicaciones han sido editados por el Grupo FITISPos-UAH con la colaboración de instituciones o empresas como la multinacional farmacéutica Pfizer, o la revista Diario Médico, y una lista de las mismas está disponibles en las siguientes páginas web: http://www2.uah.es/traduccion ; http://www.fitispos.com.es.

Finalmente, como modo de difusión de los resultados de nuestras propias investigaciones así como las de otros investigadores y profesionales de nuestro campo de trabajo, mencionamos la organización de 5 ediciones del Congreso Internacional sobre Traducción e Interpretación en los Servicios Públicos de la UAH así como de una larga lista de jornadas, seminarios o cursos relacionados con el tema desde el año 1995 con el $I$ Encuentro Internacional de Traducción con el lema "Cultura sin fronteras". El objetivo siempre fue acercar a la comunidad profesional y académica, al tema de la comunicación intercultural y la traducción e interpretación en particular como fuente de diálogo en sociedades multilingües y multiculturales. Algunas de las publicaciones más representativas, aunque no las únicas, son: Nuevas Necesidades para nuevas realidades / New Needs for New Realities (2002), Traducción como mediación entre lenguas y culturas / Translation as Mediation or how to Bridge Linguistic and Cultural Gaps (2005); Investigación y práctica en Traducción e Interpretación en los Servicios Públicos: desafíos y alianzas / Research and Practice in Public Service Interpreting and Translation: Challenges and Alliances (2008); Crossing Borders in Community Interpreting. Definitions and Dilemas (2008); Avances y retos en la Traducción e Interpretación en los Servicios Públicos / Challenges Topics in Public Service Interpreting and Translating (2009); Traducción e Interpretación en los Servicios Públicos en un mundo INTERcoNEcTado (TISP en INTERNET) / Public Service Interpreting and Translation in a Wild Wired World (PSIT in www) (2011); Traducción e Interpretación en los Servicios Públicos en el siglo XXI. Avanzando hacia la unidad en medio de la globalización (2011); La comunicación en el ámbito médico-sanitario / Communicating in the Healthcare Setting (2013)

En la misma línea, y para dar una mayor accesibilidad a las investigaciones de nuestros alumnos, nos proponemos insistir más en la presentación de trabajos derivados de los TFM en congresos internacionales y publicación de artículos en actas y revistas de investigación como las que ya se han reseñado, animándoles a seguir investigando. 


\section{Conclusiones}

El programa del Máster Universitario en Comunicación Intercultural, Interpretación y Traducción en los Servicios Públicos de la Universidad de Alcalá une formación, investigación y práctica en un contexto multidisciplinar, multilingüe y multicultural y en consonancia con las nuevas tendencias en formación. En esta ocasión nos hemos centrado en la investigación llevada a cabo por los propios alumnos como complemento a su formación. El análisis de sus características nos muestra que es una investigación que, a pesar de sus puntos débiles, supone también una contribución importante tanto para la formación como para la práctica.

En este sentido, se han presentado acciones que se han y se están llevando a cabo para dar visibilidad y rentabilidad a la misma, así como acercar a los profesionales, investigadores y alumnos mediante una importante labor de difusión y acceso al público a investigaciones específicas y de utilidad que están muy poco conocidas o prácticamente desconocidas.

\section{Referencias bibliográficas}

AGORA. "Transnational Placement Scheme for Translation Students." [https://www.academic-projects.eu/agora/Pages/project_es.aspx].

Building Mutual Trust. "A Frame Work Project For Implementing EU Common Standards In Legal Interpreting And Translating”. [http://www.lr.mdx.ac.uk/mutual-trust/].

Cohen, L., Manion, L., \& Morrison, K. 2000. Research methods education (5th ed.). Londres y Nueva York: Routledge.

Grubbs et al. 2006. "Effect of Awareness of Language Law on language Access in the health Care Setting". Journal of General Internal Medicine, 21 (7). 683-688, July 2006.

Hale, S. 2010/2007. La interpretación comunitaria. La interpretación en los sectores jurídico, sanitario y social. Granada: Comares. Título original: Community Interpreting. Basingstoke: Palgrave/Macmillan, 2007. Traducción de R. Cobas Álvarez y C. Valero Garcés.

Jacobs, E. et al. 2004. "What a Difference an Interpreter Can Make: Health Care Experiences of Uninsured with Limited English Proficiency". American Journal of Public Health .May. 866-869,

Morales, M. E. et al. 2006 "The Impact of Interpreters on Parents' Experiences with Ambulatory Care for Their Children”. Medical Care Research and Review. 63 (1).

OPTIMALE, Erasmus Network for Professional Training of Translators [http://www.translator-training.eu/].

QUALETRA, Quality in Legal Translation [http://www.eulita.eu/qualetra].

SIGTIPS, "Special Interest Group on Translation and Interpreting in Public Services" [http://www.celelc.org/archive/working-groupreports/sigtips_final_report/index.html].

Sturby, A. 2007. "Avoiding Burnout of Bilingual Employees". The Race Equity Project, September.

Valero Garcés, C. 2008. "Primeros pasos en investigación en TISSPP. ¡Esa difícil tarea de abrir camino!"’. En Carmen Valero- Garcés \& Carmen Pena. (eds.). Investigación y Práctica en Traducción e Interpretación en los Servicios Públicos: Desafíos y Alianzas / Research and Practice in Public Service Interpreting and Translation: Challenges and Alliances. Alcalá de Henares: Publicaciones Universidad de Alcalá, Madrid. 
Valero Garcés. C. 2013a. "Comunicación con población extranjera en el ámbito sanitario. Iniciación a una base de datos sobre artículos de investigación”. Panace@, 15 (37): 80-86.

Valero Garcés, C. 2013b. "Formación de traductores e intérpretes en una sociedad multicultural. El programa de la Universidad de Alcalá, Madrid". Cuadernos de ALDEEU, 25: 215- 238. 